\title{
HMGB1 knockdown effectively inhibits the progression of rectal cancer by suppressing HMGB1 expression and promoting apoptosis of rectal cancer cells
}

\author{
ZHIWEI WANG ${ }^{1,2}$, XIAOYAN WANG ${ }^{3}$, JIANTIAN LI $^{2}$, CHENG YANG $^{2}$, \\ ZHIYUAN XING ${ }^{1,2}$, RUIYUN CHEN ${ }^{2}$ and FEI XU ${ }^{2}$ \\ ${ }^{1}$ Qingdao Medical College, Qingdao University; ${ }^{2}$ Department of Gastrointestinal and Anorectal Surgery; \\ ${ }^{3}$ Healthcare Ward, Qingdao Central Medical Group, Qingdao, Shandong 266042, P.R. China \\ Received July 3, 2015; Accepted May 3, 2016
}

DOI: $10.3892 / \mathrm{mmr} .2016 .5340$

\begin{abstract}
Rectal cancer is a malignant gastrointestinal tumor, which is associated with high morbidity and mortality. High-mobility group protein 1 (HMGB1) is widely present in the nucleus of eukaryotic cells, and is highly conserved between humans and rodents. Recently, HMGB1 has been reported to be involved in the progression and metastasis of human cancer; however, its role in the development and metastasis of human rectal cancer remains unclear. The present study detected the expression levels of HMGB1 in pathological specimens from patients with clinically identified rectal cancer using immunohistochemistry and western blotting. The results demonstrated that HMGB1 was highly expressed in samples from patients with rectal cancer. The positive rate of HMGB1 in rectal cancer tissues was $96.08 \%$ (49/51), which was significantly higher compared with $3.92 \%$ (2/51) in normal tissues. In addition, western blotting indicated that HMGB1 was distributed and located not only in the nucleus, but also in the cytoplasm of colorectal cancer cells. HMGB1-specific short hairpin (sh)RNA was used to silence the endogenous expression of HMGB1 in colorectal cancer cells. A functional assay demonstrated that knockdown of endogenous HMGB1 expression significantly inhibited the proliferation of SW620 and Colo320 cells. Furthermore, western blotting revealed that knockdown of endogenous HMGB1 expression contributed to activation of caspase- 3 and the substrate poly (ADP-ribose) polymerase. The expression levels of B-cell lymphoma 2 (Bcl-2) and Bcl-2-associated X protein (Bax) were also detected by western blotting. As expected, decreased levels of Bcl-2 and increased levels of Bax were detected in the HMGB1 shRNA-transfected colorectal cancer cells, and the Bax/Bcl-2
\end{abstract}

Correspondence to: Dr Fei Xu, Department of Gastrointestinal and Anorectal Surgery, Qingdao Central Medical Group, 127 Siliunan Road, Qingdao, Shandong 266042, P.R. China

E-mail: xufeixfly@163.com

Key words: rectal cancer, high-mobility group protein 1, cell apoptosis, caspase-3 ratio was increased in HMGB1 shRNA-transfected cells. These data indicated that HMGB1 may act as an oncogene in rectal cancer, and knockdown of endogenous HMGB1 expression may significantly inhibit the proliferation of colorectal cancer cells and promote apoptosis of tumor cells. Further research regarding the mechanisms underlying the effects of HMGB1 on the progression of rectal cancer may provide novel targets for the treatment of rectal cancer, and provide a theoretical reference for clinical treatment.

\section{Introduction}

Rectal cancer is one of the most common types of gastrointestinal malignancy, and refers to tumors that occur between the dentate line and the rectosigmoid junction (1). The median age of patients with rectal cancer is $\sim 45$ years; however, the incidence in young people has begun to increase. Rectal cancer is easily diagnosed by a digital rectal examination and sigmoidoscopy (2-4). However, since the cancer is located deep within the pelvic cavity, and due to the key anatomical differences between the rectum and colon, the management and treatment of rectal cancer is significantly difficult, particularly in the curative setting (5). Rectal tumors are not easily completely removed by surgery alone, thus leading to a high recurrence rate in patients with rectal cancer (6), which is a common cause of cancer-associated mortality worldwide. Although great progress has been made regarding the diagnosis and treatment of rectal cancer, the prognosis remains poor for patients with distant metastasis of rectal cancer (7). Clinically, micrometastases occur in the majority of patients with rectal cancer prior to undergoing radical operation, and are the direct cause of metastasis and recurrence post-surgery in patients with rectal cancer $(2,5)$. Therefore, it is essential to further explore the mechanisms underlying the development and progression of rectal cancer and to clarify its biological characteristics, in order to develop more effective therapeutic targets that may improve the prognosis of patients with rectal cancer.

High-mobility group protein 1 (HMGB1) is a recently discovered oncogene, with a molecular weight of $\sim 30 \mathrm{kD}(8)$. HMGB1 is a non-histone chromosomal protein, which is widely present in the nucleus of eukaryotic cells. It is named for its 
fast migration velocity in polyacrylamide gel electrophoresis (PAGE) $(9,10)$. HMGB1 is regarded as a proinflammatory factor that is secreted from the nucleus to the extracellular space. In addition, HMGB1 is highly conserved between humans and rodents, and the amino acid sequences are $<99 \%$ homologous. Notably, the amino acid sequence of HMGB1 is exactly the same between rats and mice. HMGB1 has two DNA-binding motifs; A box and B box, which execute two major functions. The HMGB1 B box exerts cytokine activity, whereas A box has an inverse role and inhibits the activity of native HMGB1. HMGB1 has various receptors, including receptor for advanced glycation end products (RAGE), Toll-like receptors (TLRs), syndecan and plasminogen, among which, RAGE and TLR4/TLR2 are the main receptors of HMGB1.

In recent years, high expression levels of HMGB1 have been detected in breast cancer (11), colon cancer $(12,13)$, lung cancer $(14,15)$, bladder cancer $(16)$, ovarian cancer (17), and other tumors (18). HMGB1 is involved in numerous processes during tumor formation and development, including unlimited cell proliferation, angiogenesis, programmed cell death evasion, and resistance to growth factor inhibitors. Previous studies have reported that elevated expression of HMGB1 is closely associated with a high incidence of tumor invasion and metastasis (19-21). However, the role of HMGB1 in human rectal cancer remains to be clarified. The present study detected the expression levels of HMGB1 in rectal cancer specimens and explored the mechanisms underlying the effects of HMGB1 on colorectal cancer cells. Understanding the mechanisms underlying rectal cancer may help to identify novel targets during the progression and metastasis of rectal cancer.

\section{Materials and methods}

Patients. A total of 51 patients with rectal cancer and 51 normal controls were included in the present study. In the rectal cancer group, 39 patients were male $(76.5 \%)$ and 12 patients were female $(23.5 \%)$. In the control group, 32 patients were male $(62.7 \%)$ and 19 patients were female $(37.3 \%)$. The median age was 60.47 years, with a range of $26-85$ years. The present study was approved by the ethics committee of Qingdao Central Medical Group (Qingdao, China), and all patients provided written informed consent. The subjects were well informed of the details of the study and signed relevant contracts prior to the study. Half of each postoperative rectal specimen was immediately fixed in $10 \%$ neutral buffered formalin solution and embedded in paraffin. The other half of each specimen was frozen and maintained at $-80^{\circ} \mathrm{C}$ until further use. The expression levels of HMGB1 were detected by immunohistochemical analysis and western blotting. Peritumoral tissue was obtained during he surgery.

Cell lines and agents. The SW620 and HCT116 colorectal cancer cell lines were obtained from the American Type Culture Collection (Manassas, VA, USA), and Colo320 colorectal cells were purchased from the Chinese Acadamy of Tumor Cell Bank (Shanghai, China). The cells were cultured in Dulbecco's modified Eagle's medium (DMEM) supplemented with $10 \%$ fetal bovine serum (FBS) (both Hyclone; GE Healthcare Life Sciences, Logan, UT, USA) at $37^{\circ} \mathrm{C}$ with $5 \% \mathrm{CO}_{2}$. The human HMGB1 short hairpin (sh)RNA and control shRNA (cat. no. TR30013) were obtained from Origene Technologies, Inc. (Rockville, MD, USA). The HMGB1 shRNA consisted of four unique 29mer shRNAs (cat. no. TG316576). Lipofectamine 2000 was used for transfection of the cells (Invitrogen; Thermo Fisher Scientific, Inc, Waltham, MA, USA).

Antibodies and small interfering (si)RNAs. Mouse monoclonal anti-HMGB1 (cat. no. ab77302) was obtained from Abcam (Cambridge, MA, USA). Rabbit polyclonal anti-caspase-3 (cat. no. 3138-100) was purchased from BioVision, Inc. (Milpitas, CA, USA). Rabbit polyclonal anti-B-cell lymphoma 2 (Bcl-2; cat. no. AP1303a-ev), mouse polyclonal anti-Bcl-2-associated X protein (Bax; cat. no. AP1302a-ev) and mouse monoclonal anti- $\beta$-actin (cat. no. P60709) were obtained from Abgent Biotech Co., Ltd. (Suzhou, China), and anti-cleaved-PARP (cat. no. ab4830; Abcam, Cambridge, MA, USA). All antibodies were used at a dilution of 1:1,000. The siRNAs were designed and synthesized by GenePharma Corp. (Shanghai, China). The HMGB1-specific siRNA sequences were as follows: HMGB1 siRNA1, sense 5'-GGAGAUCCU AAGAAGCCGATT-3', antisense 5'-UCGGCUUCUUAGGAU CUCCTT-3'; HMGB1 siRNA2, sense 5-CUGCGAAGCUGA AGGAAAATT-3', antisense 5'-UUUUCCUUCAGCUUC GCAGTT-3'; and HMGB1 siRNA3, sense 5'-GGGAGGAGC AUAAGAAGATT-3', antisense 5'-UUCUUCUUAUGCUCC UCCCTT-3'. Negative control (N.C.) siRNA was purchased from GenePharma Corp. (cat. no. A01004), which was purified by HPLC and the unpaired single chains were truly removed.

Transfection. The SW620 cells $\left(1 \times 10^{6}\right.$ cells/well) were plated into a 48-well plate. After 8 h, $200 \mathrm{ng}$ of three pairs of HMGB1-specific siRNA and $2 \mu$ l Lipofectamine 2000 (Thermo Fisher Scientific, Inc.), according to the manufacturer's protocol. The mixture was added into the medium of human colorectal cancer cells and cultured for $48 \mathrm{~h}$.

MTT assay. A 3-(4,5-dimethyl-2-thiazolyl)-2,5-diphenyl-2H-tetrazolium bromide (MTT) assay was used to determine the proliferation of SW620 and Colo320 cells. The SW620 and Colo320 colorectal cancer cells were plated into 48 -well plates at a density of $2 \times 10^{4}$ cells/well. After $6 \mathrm{~h}$, the cells were transfected with HMGB1 shRNA or N.C. shRNA using Lipofectamine 2000, and the cells were cultured for 24, 48, 72 and $96 \mathrm{~h}$. Subsequently, $10 \mu \mathrm{l} 5 \mathrm{mg} / \mathrm{ml}$ MTT (Sigma-Aldrich, St. Louis, MO, USA) was added to the cells for $4 \mathrm{~h}$ at $37^{\circ} \mathrm{C}$. The medium was gently aspirated and $100 \mu \mathrm{l}$ dimethyl sulfoxide was added and the cells were incubated for $15 \mathrm{~min}$. Absorbance was measured at a wavelength of $490 \mathrm{~nm}$ using a microplate reader. The cells transfected with N.C. shRNA were used as negative controls and the experiment was repeated three times.

Immunohistochemical analysis. Immunohistochemical staining was performed to detect the expression and distribution of HMGB1 in rectal cancer tissues. Briefly, the sections were dewaxed in xylene for 5-10 min and were exposed to a graded alcohol solution at concentrations of 100, 95, 90, 80 and 70\%, respectively, for 3-5 min for rehydration. Following this, the 
sections were placed in distilled water for $3 \mathrm{~min}$. Finally, the sections were sealed with neutral gum and incubated overnight with a primary anti-HMGB1 antibody at room temperature. The specimens were then washed three times with phosphatebuffered saline for $5 \mathrm{~min}$, and were incubated with goat anti-mouse secondary antibody for $1 \mathrm{~h}$ at room temperature. The stained slides were visualized and images were captured using a Nikon Eclipse Ti microscope (Nikon Corporation, Tokyo, Japan). The positivity of immunostaining was identified in eight different fields at 400x magnification.

Western blotting. For total protein, the samples and cells were washed in phosphate-buffered saline and were lysed in radioimmunoprecipitation assay buffer $[50 \mathrm{mmol} / 1$ of Tris, $1 \%$ $\mathrm{NP}-40,150 \mathrm{mmol} / \mathrm{l}$ of $\mathrm{NaCl}, 1 \mathrm{mmol}$ of EDTA, $1 \%$ sodium dodecyl sulfate (SDS) and $0.25 \%$ SDC]. When assessing cytoplasmic or nuclear proteins, a nuclear protein extraction kit (Beyotime Institute of Biotechnology, Beijing, China), according to the manufacturer's protocol. The concentration of whole proteins in the lysates was determined using the Bradford assay (Beyotime Institute of Biotechnology). Protein samples $(10 \mu \mathrm{g})$ were separated by $10 \%$ SDS-PAGE and were then transferred electrophoretically onto a nitrocellulose membrane. Subsequently, the membrane was blocked with 5\% bovine serum albumin (Beyotime Institute of Biotechnology) in Tris-buffered saline containing Tween $(50 \mathrm{mmol} / \mathrm{l}$ Tris- $\mathrm{HCl}$, $150 \mathrm{mmol} / \mathrm{l} \mathrm{NaCl}$ and $0.1 \%$ Tween). The membranes were incubated with primary antibodies overnight at $4^{\circ} \mathrm{C}$. The membranes were then incubated with horseradish peroxidase-conjugated goat anti-mouse (cat. no. sc-2031; 1:1,000) or goat anti-rabbit immunoglobulin G (cat. no. sc-2004; 1:1,000; each from Santa Cruz Biotechnology, Inc., Santa Cruz, CA, USA) secondary antibodies for $1 \mathrm{~h}$ at room temperature. The resulting bands were detected using an enhanced chemiluminescence western blotting detection system (Thermo Fisher Scientific, Inc.), according to the manufacturer's protocol. $\beta$-actin was used as an internal reference. The gray value of the protein band was analyzed using ImageJ version $1.38(\mathrm{NIH}$, Bethesda, MD, USA).

Statistical analysis. Statistical analyses were performed using SPSS 20.0 statistical software (IBM SPSS, Armonk, NY, USA). Clinical indicators were compared using $\chi^{2}$ test. The data are presented as the mean \pm standard deviation. $\mathrm{P}<0.01$ was considered to indicate a statistically significant difference.

\section{Results}

HMGB1 is highly expressed in samples from patients with rectal cancer, as detected by immunohistochemical analysis. A total of 51 patients and 51 normal controls were studied in the present study; the median age was 60.47 years, with a range of 26-85 years. HMGB1 expression was detected by immunohistochemical analysis, and images of the specimens were captured (original magnification, x400; Fig. 1). HMGB1 protein was predominantly located in the cytoplasm and nucleus of rectal tumor cells, with control tissues exhibiting no positive staining for HMGB1. Positive HMGB1 expression appeared as brown cytoplasmic and nuclear staining. The whole panel of rectal cancer and normal tissues was tested.

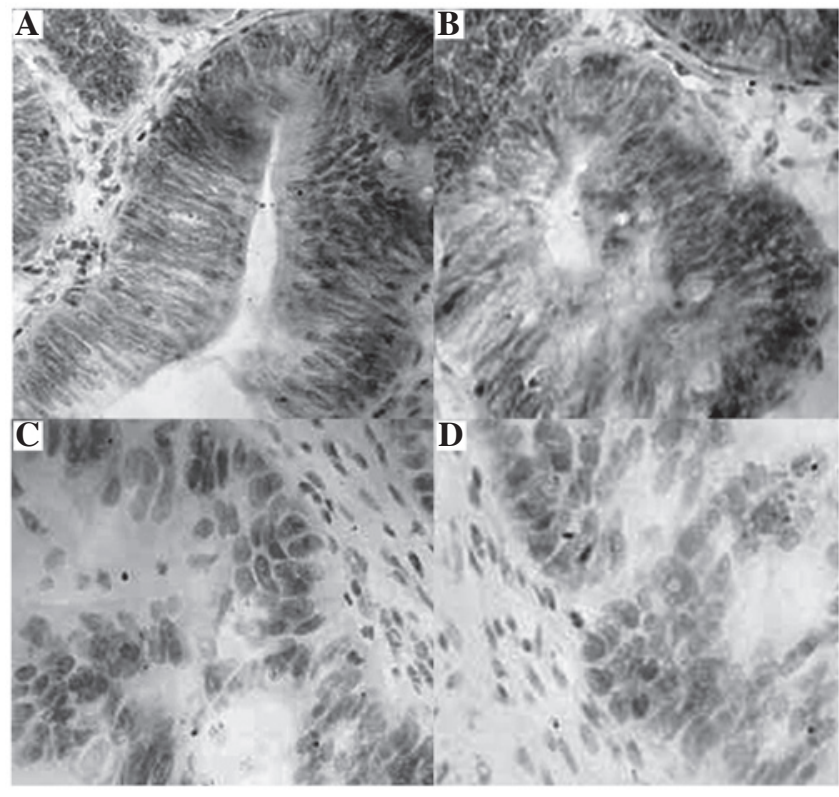

Figure 1. Immunohistochemical detection of high-mobility group protein 1 (HMGB1) in rectal cancer and normal tissues. Paraffin-embedded samples were analyzed by immunohistochemistry. (A and B) Rectal cancer tissues. HMGB1 protein was mainly located and expressed in the cytoplasm and nucleus of rectal tumor cells. (C and D) Normal tissues, which were used as negative controls. Negative expression of HMGB1 was detected in the control tissues (original magnification, 400x).

Table I. HMGB1 expression in rectal cancer and normal tissues.

\begin{tabular}{lcc}
\hline Group & Normal tissues & Rectal cancer tissues \\
\hline HMGB1 & $2(3.92 \%)$ & $49(96.08 \%)$ \\
P-value & $<0.001$ & \\
\hline
\end{tabular}

HMGB1, high-mobility group protein 1 .

As presented in Table I, the positive rate of HMGB1 expression was $96.08 \%(49 / 51)$ in rectal cancer tissues, which was significantly higher than in normal tissues $(3.92 \% ; 2 / 51)$.

Western blot analysis of HMGB1 expression in rectal cancer tissues. In order to further detect the expression levels of HMGB1 in rectal cancer, rectal cancer tissues, peritumoral tissues and normal control tissues (three specimens each) underwent western blotting. As shown in Fig. 2A, the expression levels of HMGB1 were markedly increased in rectal cancer tissues compared with in peritumoral or normal control tissues, which was consistent with the immunohistochemical results. $\beta$-actin was used as an internal reference. As shown in Fig. $2 \mathrm{~B}$, the data were normalized to $\beta$-actin and expressed as mean \pm standard deviation. The expression levels of HMGB1 were significantly higher in rectal cancer tissues compared with in peritumoral tissues $(\mathrm{P}<0.01)$ or normal control tissues $(\mathrm{P}<0.01)$.

HMGB1 is located in the cytoplasm and nucleus of colorectal cancer cells. Three colorectal cancer cell lines were selected 


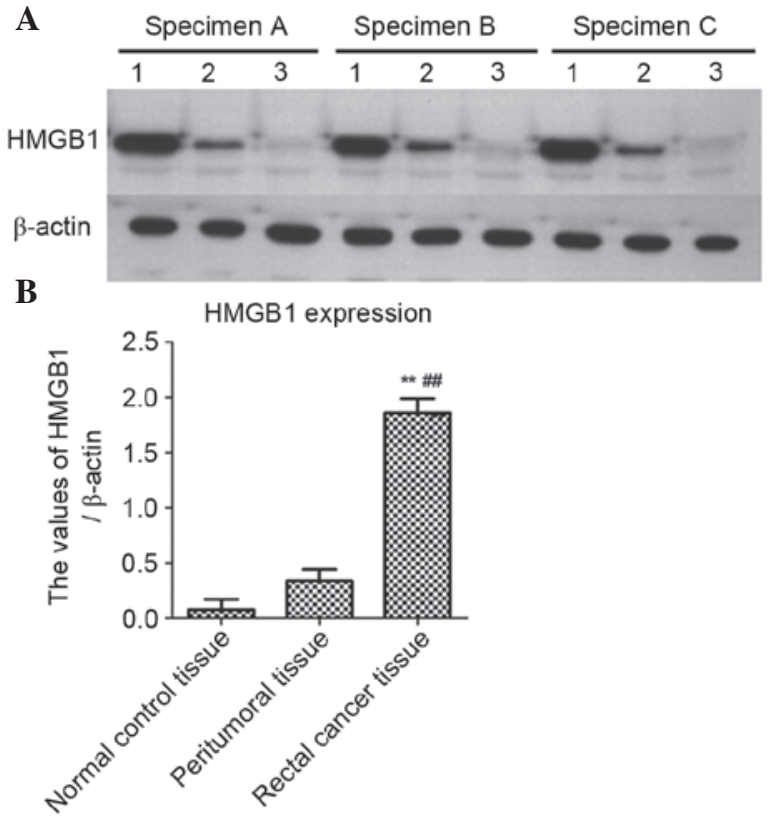

Figure 2. Western blot analysis of high-mobility group protein 1 (HMGB1) expression in rectal cancer tissues. (A) HMGB1 expression was detected in three independent specimens by western blotting. $\beta$-actin was used as an internal reference. (B) Histogram of HMGB1 expression. Gray values of the bands were captured and analyzed by ImageJ software. Data are presented as the mean \pm standard deviation. ${ }^{* *} \mathrm{P}<0.01$, compared with control tissues; ${ }^{\# \#} \mathrm{P}<0.01$, compared with peritumoral tissues. Specimens A, B and C are three independent specimens. 1, rectal cancer tissue; 2, peritumoral tissue; 3 , normal control tissue.
A

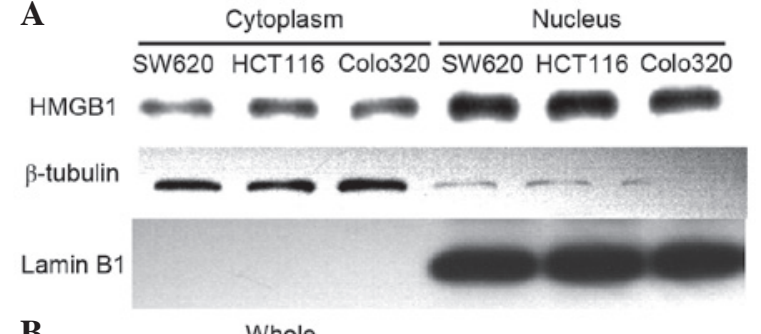

B

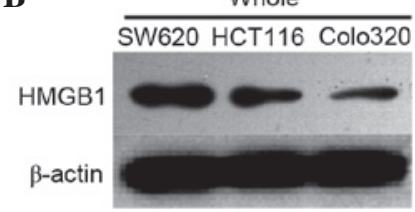

Figure 3. High-mobility group protein 1 (HMGB1) is located in the cytoplasm and nucleus of colorectal cancer cells. (A) The SW620, HCT116 and Colo320 human colorectal cancer cell lines were plated into $6-\mathrm{cm}$ dishes. After $8 \mathrm{~h}$, the cytoplasmic and nuclear proteins were extracted and whole protein concentration was determined using the Bradford assay. The expression levels of cytoplasmic and nuclear HMGB1 were determined by western blotting. (B) Cells $\left(1 \times 10^{6}\right.$ cells/well) were plated into a 48 -well plate. After $8 \mathrm{~h}$, cell lysates were prepared for western blotting. HMGB1 expression was detected by western blotting in SW620, HCT116 and Colo320 cells.

as a cell model. Western blotting was preformed to detect the expression of HMGB1 in Colo320, SW620 and HCT116 cells (Fig. 3). In order to detect the location of HMGB1, cytoplasmic and nuclear proteins were extracted, according to the kit protocol. $\beta$-tubulin was used as a cytoplasmic marker and Lamin B1 was used as a nuclear marker. The results demon-

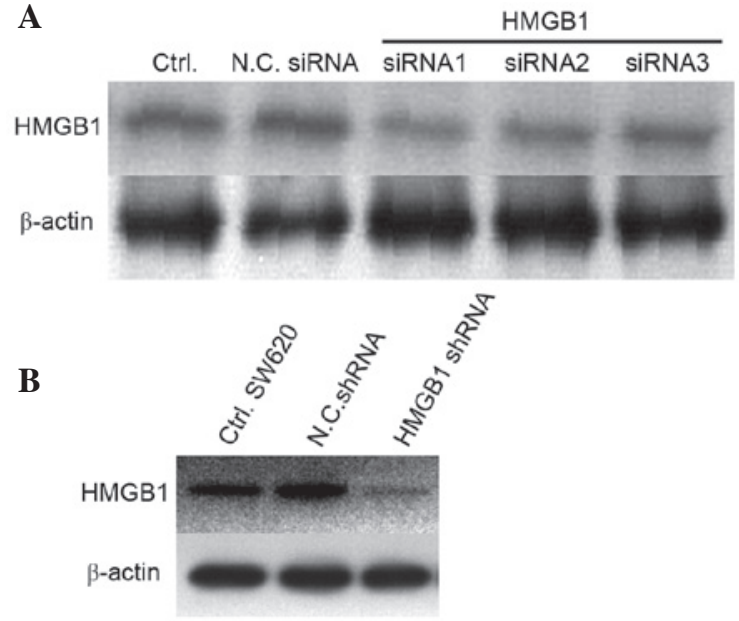

Figure 4. High-mobility group protein 1 (HMGB1) expression is successfully silenced by HMGB1-specific short hairpin (sh)RNA. SW620 cells (1x10 $10^{6}$ cells/well) were plated into a 48 -well plate. (A) Three pairs of HMGB1-specific small interfering (si)RNA were transfected into human colorectal cancer cells. (B) HMGB1 shRNA and negative control (N.C.) shRNA were used to knockdown the endogenous expression of HMGB1 in SW620 cells. After 48 h, interference efficiency was determined by western blotting. Untreated cells (Ctrl), N.C. shRNA- or N.C. siRNA-transfected SW620 cells were considered negative controls.

strated that HMGB1 was mainly located in the nucleus, but was also partly distributed in the cytoplasm of colorectal cancer cells. These findings are consistent with the results of the immunohistochemical analysis.

HMGB1 expression is successfully silenced by $H M G B 1$-specific shRNA. In order to further confirm the role of HMGB1 in cancer cells, RNA interference technology was used to knockdown the expression of HMGB1 in SW620 cells. Three siRNA pairs specific to HMGB1 were generated to interfere with endogenous HMGB1 expression in SW620 cells; however, the knockdown efficiency was not significantly altered compared with that of the N.C. siRNA (Fig. 4A). Therefore, HMGB1-specific shRNA was used to knockdown the endogenous expression of HMGB1 in SW620 cells. As shown in Fig. 4B, transfection with HMGB1-shRNA for $48 \mathrm{~h}$ effectively downregulated the endogenous expression of HMGB1, as determined by western blotting.

Knockdown of endogenous HMGB1 expression inhibits the proliferation of colorectal cancer cells. The present study aimed to determine whether knockdown of endogenous HMGB1 expression would affect the proliferation of colorectal cancer cells. Therefore, two cell lines were used as a cell model and an MTT assay was conducted to detect the proliferation of cancer cells transfected with HMGB1 shRNA. As shown in Fig. 5, the HMGB1 shRNA-transfected SW620 cells had much lower proliferative ability compared with the untreated or N.C. shRNA-transfected cancer cells. In addition, the survival rate of HMGB1 shRNA-transfected Colo320 cells was determined by MTT assay. Knockdown of endogenous HMGB1 expression inhibited the proliferation of Colo320 cells, compared with those transfected with N.C. shRNA; however, the results demonstrated that SW620 cells were more appropriate for transfection with shRNA. 



Figure 5. Knockdown of endogenous high-mobility group protein 1 (HMGB1) expression inhibits the proliferation of colorectal cancer cells. (A) SW620 cells and (B) Colo320 cells were transfected with HMGB1-specific short hairpin (sh)RNA and negative control (N.C) shRNA using Lipofectamine 2000. The cells were cultured for 24, 48, 72 and $96 \mathrm{~h}$. A 3-(4,5-dimethyl-2-thiazolyl)-2,5-diphenyl-2H-tetrazolium bromide (MTT) assay was used to determine the proliferation of SW620 and Colo320 cells. Cells transfected with N.C. shRNA were used as negative controls. The experiment was repeated three times, and all of the data are presented as the mean \pm standard deviation. ${ }^{*} \mathrm{P}<0.05,{ }^{* *} \mathrm{P}<0.01$ compared with the N.C. shRNA-transfected cells. OD, optical density.

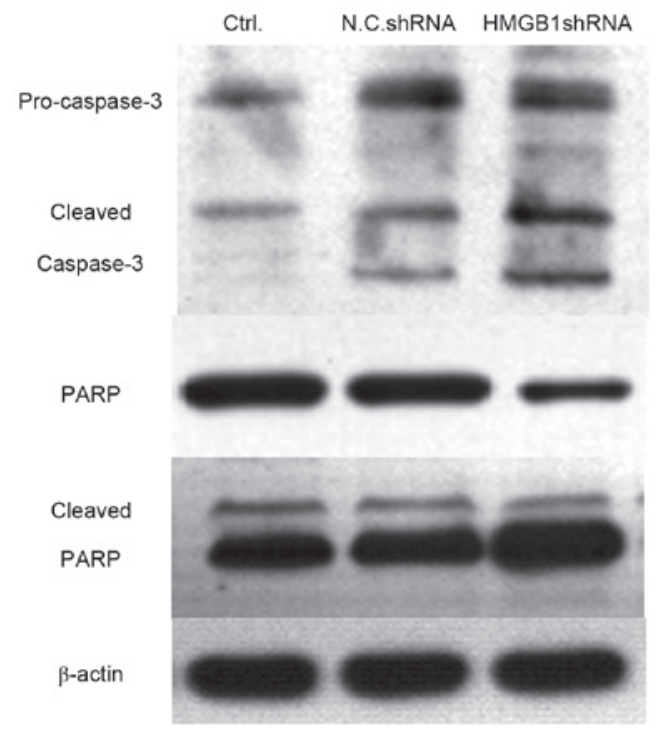

Figure 6. Knockdown of endogenous high-mobility group protein 1 (HMGB1) expression activates caspase-3 and its substrate poly (ADP-ribose) polymerase (PARP). SW620 cells $\left(5 \times 10^{5}\right.$ cells/well) were plated into 48 -well plates and were transfected with HMGB1-specific short hairpin (sh)RNA for $48 \mathrm{~h}$. The expression levels of pro-caspase-3, cleaved caspase-3, PARP and cleaved PARP were detected by western blotting. $\beta$-actin was used as an internal reference. Ctrl, control untreated cells; N.C., negative control.

Knockdown of endogenous HMGB1 expression activates caspase-3 and its substrate poly (ADP-ribose) polymerase $(P A R P)$. The present study indicated that knockdown of HMGB1 expression significantly inhibited the proliferation of SW620 and Colo320 cells. Subsequently, the present study aimed to determine whether cell apoptosis was induced in HMGB1 shRNA-transfected cells. Caspase-3 is the executor of cell apoptosis, and the activation of caspase- 3 and its substrate PARP were detected using western blotting. As shown in Fig. 6, the expression levels of cleaved capspase-3 were markedly enhanced in HMGB1 shRNA-transfected SW620 cells, compared with in the untreated or N.C. shRNA-transfected cells. Furthermore, the expression levels of PARP were decreased, whereas the levels of cleaved PARP were upregulated in HMGB1 shRNA-transfected SW620 cells. These data suggest that transfection with HMGB1 shRNA may significantly increase cell apoptosis in colorectal cancer cells.
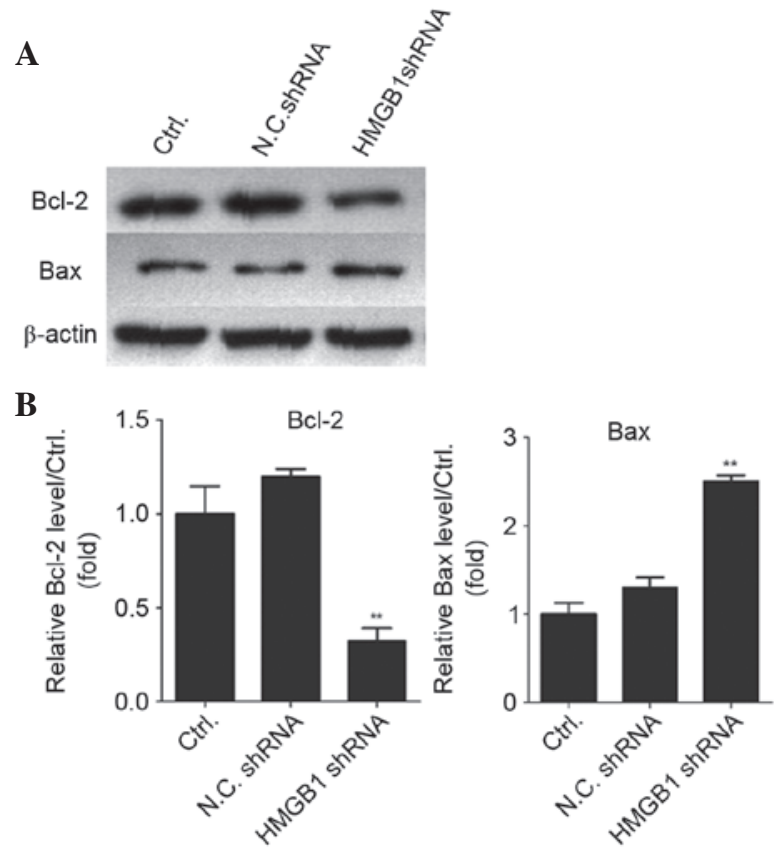

Figure 7. Knockdown of high-mobility group protein 1 (HMGB1) expression contributes to activation of cell apoptosis-associated proteins. (A) SW620 human cancer cells $\left(3 \times 10^{5}\right.$ cells/well) were plated into 48 -well plates and were transfected with HMGB1-specific short hairpin (sh)RNA for $48 \mathrm{~h}$. The expression levels of B-cell lymphoma 2 (Bcl-2) and Bcl-2-associated X protein (Bax) were detected by western blotting. $\beta$-actin was used as an internal reference. (B) Histogram of relative Bcl-2 and Bax expression. Western blotting results were analyzed using ImageJ. ${ }^{* *} \mathrm{P}<0.01$, compared with negative control (N.C.) shRNA-transfected cells. Ctrl, control.

Knockdown of HMGBI contributes to the activation of cell apoptosis-associated proteins. The present study also detected the expression levels of the cell apoptosis-associated proteins Bcl-2 and Bax. Bax is an apoptosis inducer, whereas $\mathrm{Bcl}-2$ is an apoptosis inhibitor. The $\mathrm{Bax} / \mathrm{Bcl}-2$ ratio reflects the situation of cell apoptosis. In the present study, the expression levels of Bax and Bcl-2 were detected by western blotting, and the results demonstrated that knockdown of HMGB1 resulted in downregulation of Bcl-2 and upregulation of Bax; therefore, the Bax/Bcl-2 ratio was increased in the HMGB1 shRNA-transfected colorectal cancer cells (Fig. 7). These results indicate that knockdown of endogenous HMGB1 
expression may activate the intrinsic mitochondrial apoptotic pathway.

\section{Discussion}

HMGB1 is a recently discovered oncogene that is associated with tumor progression and metastasis. It has previously been reported that HMGB1, through interactions with RAGE and TLRs, induces inflammation and promotes cancer progression (22). In addition, it has been demonstrated that HMGB1 may induce autophagy or apoptosis in cancer cells, depending on its redox status (23). Flohr et al (10) analyzed the expression patterns of HMGB1 in 13 breast cancer samples, and northern blot analyses of the $1.4 \mathrm{~kb}$ and $2.4 \mathrm{~kb}$ transcripts of HMGB1 revealed a strong intertumoral variation by a factor of 8.5 and 14.5, respectively, indicating that HMGB1 may be a marker of considerable clinical interest. The role of HMGB1 has been discussed in several types of cancer, including breast cancer (24), colorectal cancer $(9,25)$, gastric cancer (26-28), non-small cell lung cancer (29), pancreatic cancer (30), head and neck cancer (31), and gallbladder cancer (32). However, the role of HMGB1 in rectal cancer remains to be elucidated. The present study collected rectal cancer specimens, and used colorectal cancer cell lines as cell models to explore the role of HMGB1 in the progression of rectal cancer. The aim of the present study was to identify novel targets in rectal cancer therapy.

In the present study, immunohistochemistry and western blotting were used to analyze the expression levels of HMGB1 in pathological specimens from patients with clinically identified rectal cancer. In addition, the distribution of HMGB1 in tumor cells was detected by western blot analysis. The results demonstrated that HMGB1 was highly expressed in specimens from patients with rectal cancer. Notably, HMGB1 was distributed and located not only in the nucleus, but also in the cytoplasm of colorectal cancer cells. These results indicated that HMGB1 may have an important role in the progression and development of rectal cancer. Consistent with these findings, Song et al (18) demonstrated that HMGB1 was overexpressed in $\sim 85 \%$ of gastric cancers, and downregulation of HMGB1 increased the proportion of cells in $\mathrm{G}_{0} / \mathrm{G}_{1}$ phase and sensitized gastric cancer cells to apoptosis via the caspase-3 pathway.

Livesey et al (33) hypothesized that direct molecular interactions between HMGB1 and TP53 in colorectal cancer regulated the balance between apoptosis and autophagy through regulation of the cytosolic localization of the reciprocal binding partner. Increased cytosolic HMGB1 was shown to enhance autophagy, whereas increased cytosolic TP53 enhanced apoptosis in colon cancer cells. In addition, Zhang et al (21) reported that knockdown of HMGB1 inhibited growth and invasion of gastric cancer cells via the nuclear factor- $\kappa \mathrm{B}$ pathway in vitro and in vivo.

In conclusion, the present study demonstrated that knockdown of endogenous HMGB1 expression in SW620 and Colo320 colorectal cancer cells was able to significantly inhibit cell proliferation. In addition, apoptosis was induced in HMGB1 shRNA-transfected colorectal cancer cells. However, the mechanism underlying the effects of HMGB1 on the regulation of cell apoptosis requires further study, and the downstream targets or receptors of HMGB1 remain to be clearly explored.

\section{References}

1. Yaffee P, Osipov A, Tan C, Tuli R and Hendifar A: Review of systemic therapies for locally advanced and metastatic rectal cancer. J Gastrointest Oncol 6: 185-200, 2015.

2. Harji DP, Griffiths B, Velikova G, Sagar PM and Brown J: Systematic review of health-related quality of life issues in locally recurrent rectal cancer. J Surg Oncol 111: 431-438, 2015.

3. Poulsen LØ, Qvortrup C, Pfeiffer P, Yilmaz M, Falkmer U and Sorbye H: Review on adjuvant chemotherapy for rectal cancer - why do treatment guidelines differ so much? Acta Oncol 54: 437-446, 2015.

4. Arezzo A, Passera R, Salvai A, Arolfo S, Allaix ME, Schwarzer G and Morino M: Laparoscopy for rectal cancer is oncologically adequate: A systematic review and meta-analysis of the literature. Surg Endosc 29: 334-348, 2015.

5. Nussbaum N and Altomare I: The neoadjuvant treatment of rectal cancer: A review. Curr Oncol Rep 17: 434, 2015.

6. Ghouti L, Pereira P, Filleron T, Humeau M, Guimbaud R, Selves J and Carrere N: Pelvic exenterations for specific extraluminal recurrences in the era of total mesorectal excision: Is there still a chance for cure? A single-center review of patients with extraluminal pelvic recurrence for rectal cancer from March 2004 to November 2010. Am J Surg 209: 352-362, 2015.

7. Guren MG, Undseth C, Rekstad BL, Brændengen M, Dueland S, Spindler KL, Glynne-Jones R and Tveit KM: Reirradiation of locally recurrent rectal cancer: A systematic review. Radiother Oncol 113: 151-157, 2014.

8. Lotze MT and DeMarco RA: Dealing with death: HMGB1 as a novel target for cancer therapy. Curr Opin Investig Drugs 4: 1405-1409, 2003.

9. Süren D, Yildirim M, Demirpençe Ö, Kaya V, Alikanoğlu AS, Bülbüller N, Yildız M and Sezer C: The role of high mobility group box 1 (HMGB1) in colorectal cancer. Med Sci Monit 20: 530-537, 2014.

10. Flohr AM, Rogalla P, Meiboom M, Borrmann L, Krohn M, Thode-Halle B and Bullerdiek J: Variation of HMGB1 expression in breast cancer. Anticancer Res 21: 3881-3885, 2001.

11. Jiao Y, Wang HC and Fan SJ: Growth suppression and radiosensitivity increase by HMGB1 in breast cancer. Acta Pharmacol Sin 28: 1957-1967, 2007.

12. Ohmori H, Luo Y and Kuniyasu H: Non-histone nuclear factor HMGB1 as a therapeutic target in colorectal cancer. Expert Opin Ther Targets 15: 183-193, 2011.

13. Moriwaka Y, Luo Y, Ohmori H, Fujii K, Tatsumoto N, Sasahira T and Kuniyasu H: HMGB1 attenuates anti-metastatic defense of the lymph nodes in colorectal cancer. Pathobiology 77: 17-23, 2010.

14. Wang C, Fei G, Liu Z, Li Q, Xu Z and Ren T: HMGB1 was a pivotal synergistic effecor for $\mathrm{CpG}$ oligonucleotide to enhance the progression of human lung cancer cells. Cancer Biol Ther 13: 727-736, 2012.

15. Liu Y, Zhang P, Wu Z, Chen J and Zhou Q: Screening of highly-expressed-HMGB1-gene human lung cancer cell lines. Zhongguo Fei Ai Za Zhi 12: 965-968, 2009 (In Chinese).

16. Yang GL, Zhang LH, Bo JJ, Huo XJ, Chen HG, Cao M, Liu DM and Huang YR: Increased expression of HMGB1 is associated with poor prognosis in human bladder cancer. J Surg Oncol 106: 57-61, 2012.

17. Chen J, Liu X, Zhang J and Zhao Y: Targeting HMGB1 inhibits ovarian cancer growth and metastasis by lentivirus-mediated RNA interference. J Cell Physiol 227: 3629-3638, 2012.

18. Song B, Song WG, Li ZJ, Xu ZF, Wang XW, Wang CX and Liu J: Effect of HMGB1 silencing on cell proliferation, invasion and apoptosis of MGC-803 gastric cancer cells. Cell Biochem Funct 30: 11-17, 2012.

19. Zhang J, Liu C and Hou R: Knockdown of HMGB1 improves apoptosis and suppresses proliferation and invasion of glioma cells. Chin J Cancer Res 26: 658-668, 2014.

20. Gong W, Wang ZY, Chen GX, Liu YQ, Gu XY and Liu WW: Invasion potential of $\mathrm{H} 22$ hepatocarcinoma cells is increased by HMGB1-induced tumor NF- $\mathrm{B}$ signaling via initiation of HSP70. Oncol Rep 30: 1249-1256, 2013.

21. Zhang J, Kou YB, Zhu JS, Chen WX and Li S: Knockdown of HMGB1 inhibits growth and invasion of gastric cancer cells through the NF- $\kappa \mathrm{B}$ pathway in vitro and in vivo. Int J Oncol 44: 1268-1276, 2014 
22. Sims GP, Rowe DC, Rietdijk ST, Herbst R and Coyle AJ: HMGB1 and RAGE in inflammation and cancer. Annu Rev Immunol 28: 367-388, 2010.

23. Tang D, Loze MT, Zeh HJ and Kang R: The redox protein HMGB1 regulates cell death and survival in cancer treatment. Autophagy 6: 1181-1183, 2010.

24. Chang BP, Wang DS, Xing JW, Yang SH, Chu Q and Yu SY: MiR-200c inhibits metastasis of breast cancer cells by targeting HMGB1. J Huazhong Univ Sci Technolog Med Sci 34: 201-206, 2014.

25. Liu W, Zhang Z, Zhang Y, Chen X, Guo S, Lei Y, Xu Y, Ji C, $\mathrm{Bi} Z$ and Wang K: HMGB1-mediated autophagy modulates sensitivity of colorectal cancer cells to oxaliplatin via MEK/ERK signaling pathway. Cancer Biol Ther 16: 511-517, 2015.

26. Zhang J, Zhang R, Lu WW, Zhu JS, Xia LQ, Lu YM and Chen NW: Clinical significance of hmgbl expression in human gastric cancer. Int J Immunopathol Pharmacol 27 : 543-551, 2014

27. Li ZJ, Song B, Liu J, Han JJ, Wang CX, Zhu YX and Xu ZF: Inhibitory effect of silencing of HMGB1 gene expression on the invasive and metastatic abilities of MGC-803 gastric cancer cells. Zhonghua Zhong Liu Za Zhi 35: 244-248, 2013 (In Chinese).
28. Zhang J, Zhu JS, Zhou Z, Chen WX and Chen NW: Inhibitory effects of ethyl pyruvate administration on human gastric cancer growth via regulation of the HMGB1-RAGE and Akt pathways in vitro and in vivo. Oncol Rep 27: 1511-1519, 2012.

29. Zhang X, Wang $H$ and Wang J: Expression of HMGB1 and $\mathrm{NF}-\kappa \mathrm{B}$ p65 and its significance in non-small cell lung cancer. Contemp Oncol (Pozn) 17: 350-355, 2013

30. Wittwer C, Boeck S, Heinemann V, Haas M, Stieber P, Nagel D and Holdenrieder S: Circulating nucleosomes and immunogenic cell death markers HMGB1, sRAGE and DNAse in patients with advanced pancreatic cancer undergoing chemotherapy. Int $\mathrm{J}$ Cancer 133: 2619-2630, 2013.

31. Wild CA, Brandau S, Lotfi R, Mattheis S, Gu X, Lang S and Bergmann C: HMGB1 is overexpressed in tumor cells and promotes activity of regulatory $\mathrm{T}$ cells in patients with head and neck cancer. Oral Oncol 48: 409-416, 2012.

32. Li ML, Wang XF, Tan ZJ, Dong P, Gu J, Lu JH, Wu XS, Zhang L, Ding QC, Wu WG, et al: Ethyl pyruvate administration suppresses growth and invasion of gallbladder cancer cells via downregulation of HMGB1-RAGE axis. Int J Immunopathol Pharmacol 25: 955-965, 2012

33. Livesey KM, Kang R, Zeh HJ III, Lotze MT and Tang D: Direct molecular interactions between HMGB1 and TP53 in colorectal cancer. Autophagy 8: 846-848, 2012. 